\title{
Article \\ Effects of Fermented Vegetable Consumption on Human Gut Microbiome Diversity-A Pilot Study
}

\author{
Kerstin Thriene $^{1,+}\left(\mathbb{0}\right.$, Sina S. Hansen ${ }^{1,+}$, Nadine Binder ${ }^{2} \mathbb{D}$ and Karin B. Michels ${ }^{1, *}$ \\ 1 Institute for Prevention and Cancer Epidemiology, Faculty of Medicine and Medical Center, \\ University of Freiburg, 79110 Freiburg, Germany; kerstin.thriene@uniklinik-freiburg.de (K.T.); \\ sina.s.ullrich@gmail.com (S.S.H.) \\ 2 Institute of General Practice/Family Medicine, Faculty of Medicine and Medical Center, \\ University of Freiburg, 79110 Freiburg, Germany; nadine.binder@uniklinik-freiburg.de \\ * Correspondence: karin.michels@uniklinik-freiburg.de \\ + These authors contributed equally to this work.
}

check for updates

Citation: Thriene, K.; Hansen, S.S.; Binder, N.; Michels, K.B. Effects of Fermented Vegetable Consumption on Human Gut Microbiome Diversity -A Pilot Study. Fermentation 2022, 8, 118. https://doi.org/10.3390/ fermentation 8030118

Academic Editor: Hiroshi Kitagaki

Received: 31 January 2022

Accepted: 4 March 2022

Published: 8 March 2022

Publisher's Note: MDPI stays neutral with regard to jurisdictional claims in published maps and institutional affiliations.

Copyright: (c) 2022 by the authors. Licensee MDPI, Basel, Switzerland. This article is an open access article distributed under the terms and conditions of the Creative Commons Attribution (CC BY) license (https:/ / creativecommons.org/licenses/by/ $4.0 /)$.

\begin{abstract}
The interest in fermented food products has been increasing in recent years. Details about their microbial composition and the effects of their consumption on the human gut microbiome are of particular interest. However, evidence regarding their potential to increase gut microbial diversity, a measure likely associated with health, is lacking. To address this, we analyzed the microbial composition of commercially available fermented vegetables using $16 \mathrm{~S}$ rRNA sequencing. We also conducted a pilot study to assess the feasibility of studying the effects of regular consumption of fermented vegetables on the gut microbiome. Six healthy male volunteers participated in a randomized crossover trial, with two two-week intervention phases. Volunteers consumed $150 \mathrm{~g} / \mathrm{d}$ of either sauerkraut or a variety of six different commercially available fermented vegetables. This study is registered at the German Clinical Trials Register (DRKS-ID: DRKS00014840). Lactobacillales was the dominant family in all fermented vegetables studied. However, the alpha diversity, richness and evenness of the microbiota differed substantially among the different products. The number of species per product varied between 20 and 95. After consumption of both sauerkraut and the selection of fermented vegetables, we observed a slight increase in alpha diversity. Specifically, the amount of the genus Prevotella decreased while the amount of Bacteroides increased after both interventions. However, these initial observations need to be confirmed in larger studies. This pilot study demonstrates the feasibility of this type of research.
\end{abstract}

Keywords: fermented food; human gut microbiota; dietary intervention; crossover study; pilot study

\section{Introduction}

Fermented foods have been part of the human diet for centuries and have been associated with various health benefits including longevity, reduced risk of metabolic and immune-mediated disease and overall health [1,2]. Daily consumption of sauerkraut over a period of six weeks has been reported to induce changes in gut microbial composition, accompanied by improved gastrointestinal symptoms in patients with irritable bowel syndrome [3]. In addition to prolonging shelf-life, fermentation also increases the nutritional value of food products - potentially beneficial metabolites including vitamins, bioactive peptides and phytochemicals are synthesized during the fermentation process [4]. For vegetables, lactic acid fermentation is a common type of fermentation. This process is for example used for the production of sauerkraut or kimchi. During the fermentation process, lactic acid bacteria metabolize carbohydrates present in the raw vegetables into lactic acid. This leads to a drop in $\mathrm{pH}$, which prevents the growth of undesirable microbes such as molds and therefore prevents mal-fermentation [5]. Lactic acid fermentation can be induced by adding salt at a concentration of approximately $2 \%$ (brine) to the shredded vegetables and creating an anaerobic environment, which favors the growth of lactic acid 
bacteria. Sauerkraut and other fermented vegetables are generally produced by adding a brine solution. While the bacterial community present in sauerkraut has been reported to establish itself within approximately four weeks of incubation and to remain stable during the fermentation process [6], long-term survival of microbes is not guaranteed during shelf-life.

With the growing recognition of the importance of the gut microbiome to human health and physiology, fermented foods have experienced a surge in popularity $[1,7]$. The composition of the human microbiota is affected by various factors including lifestyle, age, and diet [8-10]. While a dysbiotic microbiome has been associated with a number of diseases such as inflammatory bowel disease, asthma, obesity, and depression, its role in the development of these disorders remains unclear [11-14]. However, these observations suggest that the gut microbiome may be a promising target for maintaining or improving human health. Fermented foods are often promoted as beneficial for gut health; however, the effect of daily consumption of fermented vegetables on the gut microbiome has not been sufficiently explored. Therefore, we investigated the bacterial content of various commercially available fermented vegetables and conducted a pilot study to evaluate the feasibility of conducting a feeding study with these products.

\section{Materials and Methods}

\subsection{Analysis of Fermented Vegetables}

We explored the bacterial composition of six commercially available fermented vegetables: beetroot with goji berries, carrot with ginger, cauliflower with curcuma, sauerkraut with cranberries (referred to as "pink sauerkraut"), daikon kimchi (all from completeorganics $\mathrm{GmbH}$, Munich, Germany), and traditional sauerkraut (Eden Frischkost- (L+)Sauerkraut, Radolfszell, Germany). We selected these products, because fermented vegetables, in particular sauerkraut, have a very high abundant microbial diversity compared to other fermented foods. To maintain consistency of microbial content as much as possible throughout our study, we chose commercially available products. The fermented vegetables we selected were the only products available that were non-pasteurized and contained live bacteria, according to the manufacturers' information. We tested the number of live lactic acid bacteria (LAB) in some batches of the fermented vegetables by cultivation on LAB-selective agar to ensure that the products contained live LAB.

According to the manufacturer's information, the traditional sauerkraut was produced by addition of starter cultures while the other fermented vegetables were produced using wild fermentation. A list of the ingredients can be found in Appendix A Table A1. Distributors were unaware of the purpose of our purchases.

\subsection{S Ribosomal RNA Gene Sequencing}

A sample of each fermented vegetable product was sent to the ZIEL Core Facility Microbiome of the Technical University Munich, Germany, for sequencing. The samples were processed according to the protocol by Reitmeier et al. (2020) [15].

\subsection{Pilot Intervention Study \\ 2.3.1. Study Design}

To assess the feasibility of a feeding study using fermented vegetables and to assess their possible effects on the composition of the human gut microbiome, a pilot dietary intervention study was conducted. This study was advertised internally at the University Hospital Freiburg. As this study was a pilot study, we included six participants to test the feasibility of conducting a large-scale study, the participants' acceptance and preference of the different fermented vegetables, and early tendencies in potentially expectable effects on the gut microbiome. We restricted this pilot study to male volunteers because men are generally more difficult to recruit for feeding studies, so that we could evaluate their compliance and the feasibility in a longer-term feeding study. We conducted a randomized crossover trial, comprising eight weeks in total with one two-week washout phase, fol- 
lowed by two two-week intervention phases, which were separated by a second two-week washout phase (Figure 1). Exclusion criteria included a history of chronic diseases, acute or chronic gastrointestinal symptoms, one or more episodes of strong diarrhea within the past two months, regular intake of oral probiotic supplements or any antibiotics within the past two months, severe dietary restraints, plans to change diet within the next two months or consumption of more than two standard drinks of alcohol per day.

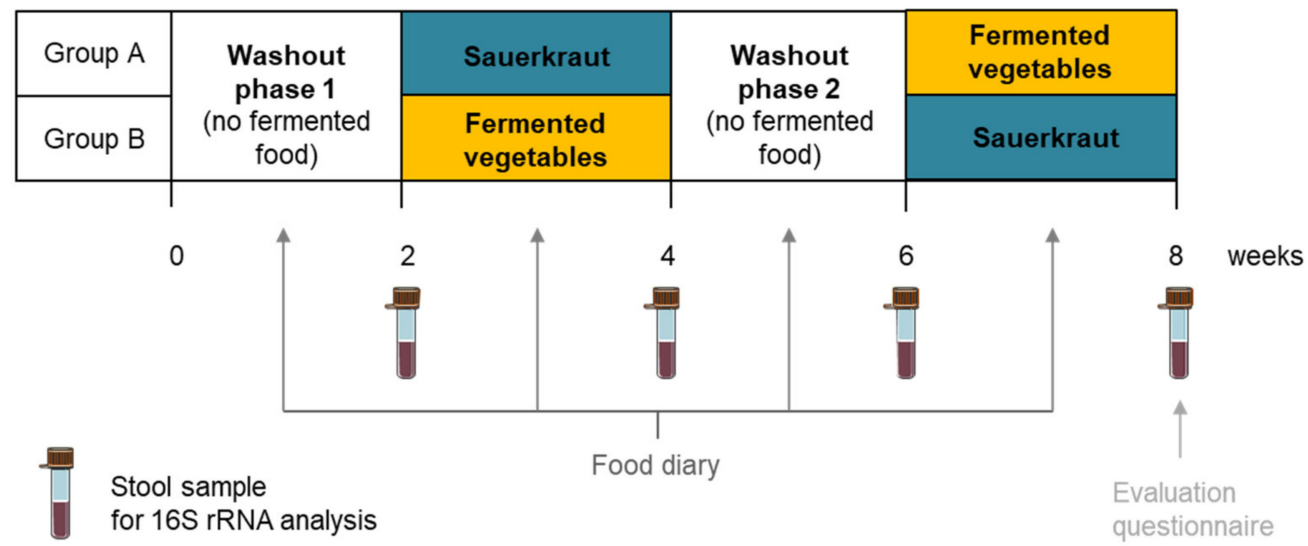

Figure 1. Study design of the randomized crossover trial, with two two-week intervention phases, each of which was preceded by a two-week washout phase. Participants consumed $150 \mathrm{~g} / \mathrm{d}$ of either sauerkraut or a variety of six different fermented vegetables. Stool samples were collected after week $2,4,6$ and 8 of this study.

The intervention consisted of consumption of $150 \mathrm{~g} /$ day of either (i) traditional sauerkraut or (ii) a selection of six different fermented vegetables: fermented beetroot, carrot, cauliflower, kimchi, pink sauerkraut, and traditional sauerkraut (as described under Section 2.1). Each product was assigned to be consumed on a certain day of the week (with traditional sauerkraut twice a week). In both parts of this study, the daily servings had to be consumed as $75 \mathrm{~g}$ portions twice a day. Participants were asked to refrain from consumption of other fermented foods, such as yogurt, kefir, or additional fermented vegetables throughout the entire study period. Stool samples were collected the day before and the day after each intervention period to establish the stool sampling process and pipeline for $16 \mathrm{~S}$ rRNA analysis. Compliance was assessed by three-day food diaries that participants kept during each study period. Participants were asked to keep diaries of adverse reactions to the intervention. At the end of this study, participants filled in a questionnaire to evaluate the feasibility of a long-term study with a comparable design, as well as their preferences regarding the intervention. This study was approved by the ethics committee of the Albert-Ludwigs-University Freiburg.

\subsubsection{Stool Sample Collection}

Stool samples were collected by participants at home in two containers ((i) native, (ii) with $96 \%$ ethanol). Participants were instructed to keep the samples in the refrigerator after collection and to return them as soon as possible (within $24 \mathrm{~h}$ ) to our lab. There, they were aliquoted and stored at $-80^{\circ} \mathrm{C}$ until subsequent analysis.

\subsubsection{Fecal Microbial DNA Isolation}

Stool samples were analyzed at the Laboratory of B. Grimbacher, University Medical Center Freiburg, Freiburg, Germany. Microbial DNA was extracted from a $2 \mathrm{~mL}$ aliquot using QIAamp DNA Stool Mini Kit (Qiagen, Hilden, Germany) following the manufacturer's instructions with modifications, similarly as described before [16-18]. Temperature of stool lysis was increased from 70 (suggested temperature in the protocol) to $95{ }^{\circ} \mathrm{C}$ for the lysis of bacteria that are known to be difficult to lyse, e.g., for Gram-positive bacteria. In a later step, $400 \mu \mathrm{L}$ supernatant (instead of suggested volume $200 \mu \mathrm{L}$ ) was pipetted into 
$15 \mu \mathrm{L}$ of proteinase $\mathrm{K}$ to which $400 \mu \mathrm{L}$ AL buffer (instead of suggested volume of $200 \mu \mathrm{L}$ ) was added followed by thorough mixing and incubation at $70{ }^{\circ} \mathrm{C}$ for $10 \mathrm{~min}$. Afterwards, a spin column was loaded twice with $400 \mu \mathrm{L}$ of the lysate.

\subsubsection{S Ribosomal RNA Gene Sequencing}

Variable (V) region 3 and 4 amplicons of 16S rRNA gene were sequenced following 16S metagenomic sequencing library protocol by Illumina [19]. 16S Amplicon PCR Forward Primer 5' TCGTCGGCAGCGTCAGATGTGTATAAGAGACAGCCTACGGGNGGCWGCAG and 16S Amplicon PCR Reverse Primer 5' GTCTCGTGGGCTCGGAGATGTGTATAAGAGACAGGACTACHVGGGTATCTAATCC were chosen as specific primers for the regions of interest of the 16S rRNA gene (V3 and V4). This reaction was set up with $2.5 \mu \mathrm{L}$ of $5 \mathrm{ng} / \mu \mathrm{L}$ concentrated DNA, $5 \mu \mathrm{L}$ 16S Amplicon PCR Forward Primer, $5 \mu \mathrm{L}$ 16S Amplicon PCR Reverse Primer and $12.5 \mu \mathrm{L} 2 \times$ KAPA HiFi HotStart ReadyMix (final volume: $25 \mu \mathrm{L}$ ). PCR products were purified using magnetic beads (Beckman Coulter, Agencourt AMPure XP-Kit). The Index PCR was set up with $5 \mu \mathrm{L}$ PCR product, $5 \mu \mathrm{L}$ Nextera Index Primer $1.5 \mu \mathrm{L}$ Nextera Index Primer 2 (different combination of primers for every sample), $25 \mu \mathrm{L} 2 \times$ KAPA HiFi HotStart ReadyMix and $10 \mu \mathrm{L}$ nuclease-free water (final volume: $50 \mu \mathrm{L}$ ). After a second PCR clean up, the size of the PCR product was visualized by gel electrophoresis. In addition, loading on a Tape Station using the D1000 High-Sensitivity Reagents from Agilent Technologies allowed quantification of the PCR product and preparation of final library by equimolar pooling of 96 samples. Library was denatured with $\mathrm{NaOH}$, diluted, mixed with 5\% PhiX control and finally loaded on MiSeq for high-throughput sequencing $(2 \times 300$ cycle V3 kit).

\subsection{Bioinformatics and Data Analysis}

All preprocessing and downstream analyses were conducted in R (v.4.1.0), an opensource free software environment for statistical computing and graphics [20]. Raw amplicon sequences were processed following the Divisive Amplicon Denoising Algorithm 2 (DADA2) workflow [21] with the DADA2 R software package (v. 1.22.0). DADA2 is able to classify sequences in the most strain-specific manner possible. Sample composition is inferred by partitioning the amplicon reads into partitions that match the error model. DADA2 derives exact amplicon sequence variants (ASVs) from the amplicon data and resolves biological differences of only 1 or 2 nucleotides. This results in an error-corrected table of the absolute abundances of the ASVs in each sample. Specifically, we followed all standard processing steps suggested by the DADA2 workflow including quality filtering, dereplication, learning the dataset-specific error model, ASV inference, chimera removal and taxonomic assignment. Within the denoise-paired function, sequences were truncated at position 260 (fermented vegetables) and 250 (stool) due to a drop-off of the quality score. Forward and reverse readings were merged, and chimeras were removed. Taxonomic assignments were obtained with the pre-trained Naïve Bayes classifier using the Silva 138.1 prokaryotic SSU taxonomic database, which specifically acknowledges the new taxonomy of Lactobacillus as characterized in [22]. A phyloseq object was created by means of the phyloseq R-package (v. 1.38.0) [23]. The species richness was determined by counting the number of different species present (observed) and alpha diversity was analyzed using Shannon diversity (richness and evenness). Beta diversity was assessed by computing weighted Bray-Curtis distances to compare microbial communities based on relative abundance. Non-metric multidimensional scaling (NMDS) was performed to compare beta diversity among groups. Relative abundances as well as alpha diversity measures were calculated using the R-package microbiome (v. 1.16.0). For computing the beta diversity, we used the R-package phyloseq. Graphics were generated using the ggplot2 R-package (v. 3.3.5). For exploring community composition, a genus-level dataset was created by agglomerating the data at the genus level, where genera that were less than $5 \%$ abundant in any of the samples (fermented vegetables) or had a relative abundance of less than $0.1 \%$ in half of the samples (stool) were aggregated into the category "Other". 
Due to the fact that this is a pilot study with a small number of participants, we focused on descriptive analyses and have deliberately refrained from any significance testing.

\section{Results}

\subsection{Bacterial Composition of Commercially Available Fermented Vegetables}

Fermented Vegetable Microbiome

Lactic acid bacteria (Lactobacillales) were detected in all analyzed fermented vegetables, among which Lactiplantibacillus was the dominant genus in most fermented vegetables displaying $38 \%$ of the total genera in a grouped analysis with all samples, followed by Lacticaseibacillus and Latilactobacillus as well as Pediococcus (Figure 2A).
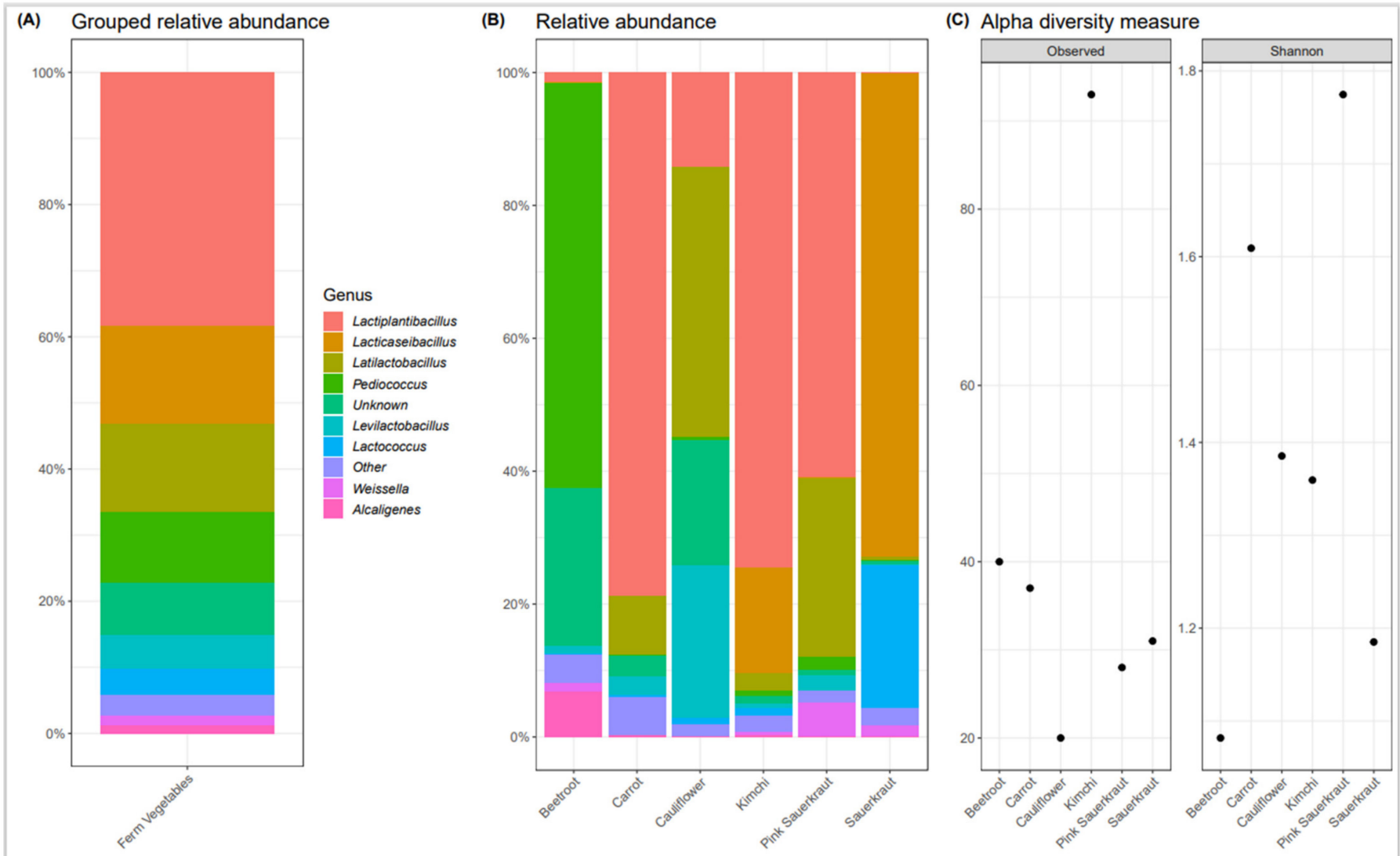

Figure 2. Bacterial composition of several commercially available fermented vegetables. Samples were analyzed by $16 \mathrm{~S}$ rRNA sequencing. (A) Grouped relative abundance of the fermented vegetables - the most abundant genera are displayed in the chart, genera that were abundant less than $5 \%$ in any of the samples were aggregated into one category ("Other"), and genera with no taxonomic annotation are categorized as "unknown"; (B) read counts (abundance) of the main bacteria species found in the fermented vegetables; $(\mathrm{C})$ alpha diversity of bacterial taxa found in the fermented vegetables.

The relative abundance of the detected genera varied between the different fermented vegetables (Figure 2B). Lactiplantibacillus was the dominant genus in carrots, kimchi and pink sauerkraut. In fermented beetroot, Pediococcus was the most dominant genus, while in the traditional sauerkraut, Lacticaseibacillus was most dominant, followed by Lactococcus. In cauliflower, Latilactobacillus followed by Levilactobacillus were the most abundant genera.

In general, alpha diversity, richness and evenness of the fermented vegetable microbiota differed substantially among the different products (Figure 2C). The number of different species (ASVs) ranged between 20 and 95 in the samples analyzed (observed 
richness). According to the Shannon index, pink sauerkraut was the most diverse sample while fermented beetroot and traditional sauerkraut displayed the lowest diversity.

\subsection{Effects of Fermented Vegetable Consumption on the Human Gut Microbiome}

Six healthy male volunteers (age: $25.5 \pm 2.9$ yrs, BMI: $24.3 \pm 1.2 \mathrm{k} \mathrm{g} / \mathrm{m}^{2}$ ) participated in this feasibility study. Compliance was very high; all participants completed all interventions. In total, $96 \%$ of the food and symptoms diaries were returned as requested. According to the food diaries, participants adhered to the intervention protocol. Four participants reported side effects, which were mainly related to gastrointestinal functions, such as flatulence and isolated episodes of diarrhea. Four participants declared their willingness to participate in a longer feeding study of this type. Three participants preferred the consumption of a selection of fermented vegetables over sauerkraut consumption. All participants returned stool samples at all requested time points. Samples stabilized in ethanol were provided by five participants, whereas native samples were provided by four participants. Further analysis was conducted using the ethanol samples for all participants except one, from whom only native samples were available.

\section{Gut Microbiome Analysis}

An increase in relative abundance was detected within the genera Bacteroides and Ruminococcus (Figure 3A). The genera Prevotella and Faecalibacterium displayed a decrease after both intervention cycles.

(A)

Relative abundance

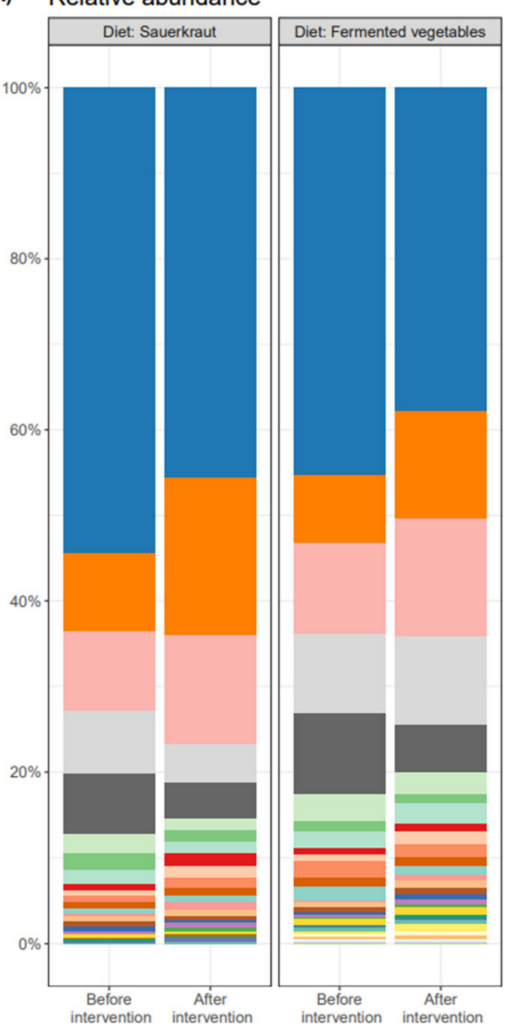

(B) Alpha diversity

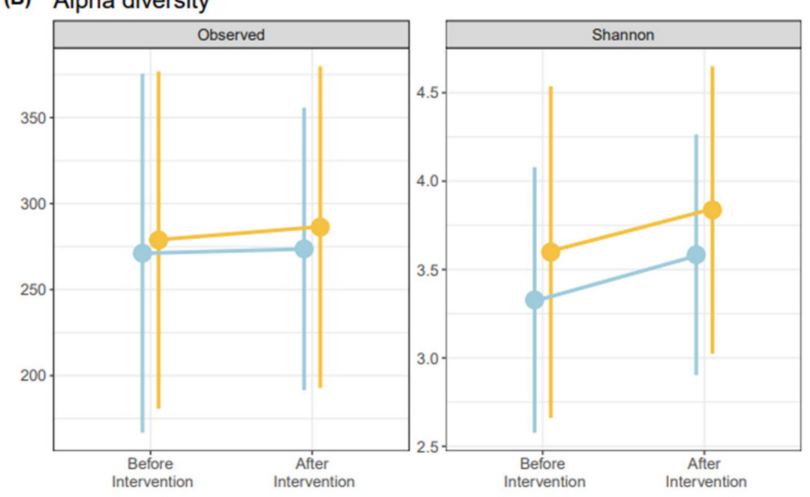

(C) Beta diversity

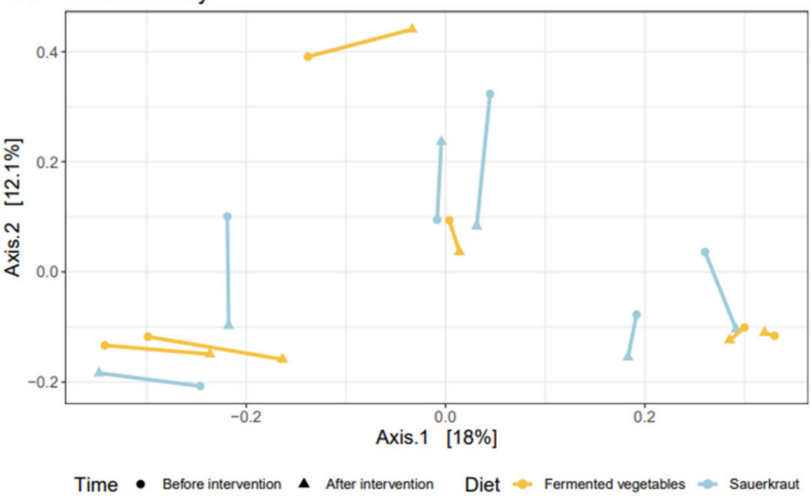

Figure 3. Human gut microbiome changes after consumption of fermented vegetables. (A) Grouped relative abundance of the main phyla found in the stool samples-the most abundant genera are displayed in the chart, genera that had a relative abundance of more than $0.1 \%$ in half of the samples were aggregated into one category ("Other"), and genera with no taxonomic annotation are categorized as "unknown"; (B) alpha diversity measures representing the diversity of bacterial taxa before and after intervention; (C) beta diversity of bacterial taxa found in the stool samples displaying each participant. 
No clear change in alpha diversity in response to either intervention was apparent according to the observed index. A slight increase in alpha diversity (the Shannon index) was observed after consumption of both sauerkraut (pre intervention: $3.31 \pm 0.74$, post intervention: $3.58 \pm 0.68$ ) and the selection of fermented vegetables (pre: $3.60 \pm 0.93$, post: $3.84 \pm 0.81$ ) (Figure 3B). Bray-Curtis dissimilarity revealed no clustering of the samples according to dietary intake and time point. For most participants, baseline and intervention samples did not differ very much, pointing towards an individualized and heterogeneous microbial composition (Figure 3C).

\section{Discussion}

In this study, the microbial composition of commercially available fermented vegetables was analyzed using $16 \mathrm{~S}$ rRNA sequencing. The effect of consumption of these vegetables on the composition of the human gut microbiome was also examined through a pilot human intervention study.

We analyzed the bacterial composition of commercially available fermented vegetables. As expected, the lactic acid bacteria family was the most represented in all fermented vegetables assessed. The genus Lactiplantibacillus was the most common in half of the fermented vegetables. Species of the Lactiplantibacillus genus are found in various fermented vegetables, especially sauerkraut and kimchi, and produce lactic acid [24]. The genera Lacticaseibacillus and Latilactobacillus are widely found in fermented foods with some species exhibiting probiotic properties. Among other genera, the genus Pediococcus is important for its preservative properties. This genus converts the sugars in fresh vegetables into lactic acid, which causes a drop in $\mathrm{pH}$ and prevents the food from spoiling [25]. The second most prominent genus detected in the sauerkraut was Lactococcus, which is made up of homofermentative species that produce lactic acid through fermentation of glucose. Many Lactococcus species are widely used to produce fermented dairy products. Another genus that was detected in the fermented vegetables, but primarily in the two sauerkraut types, was Weissella. Species of this genus are found in various fermented foods, many of them producing exopolysaccharides that are involved in adhesion to surfaces and influence the viscosity and structure of the respective fermented food [26].

The commercially available fermented vegetables that were analyzed in this study contain a variety of different microbial components, of which many species have been described to display probiotic effects. According to Hill et al., probiotics are defined as live microorganisms that are intended to have health benefits when administered in adequate amounts [27]. However, the exact mechanisms that explain the effects each species has on the human host are still not fully understood. For example, it has remained unclear whether the live bacteria consumed adhere to the intestinal mucosa and persistently populate the human gut or whether these products have to be consumed on a regular basis to enrich the host gut flora. In comparison, fermented milk products also contain probiotic bacteria, but display a less complex microbial composition. Traditional yogurt contains Lactobacillus delbrueckii subsp. bulgaricus and Streptococcus thermophiles. Often other LAB or bacteria from the genera Bifidobacterium are added to promote probiotic effects [28,29]. Other fermented dairy products such as kefir, a slightly carbonated viscous fermented milk drink, contain a more complex community of bacteria and yeasts when traditionally produced. However, in industrial production, instead of spontaneous fermentation or fermentation with kefir grains, standardized starter cultures are often used to achieve consistent product quality and suppress undesirable fermentation processes, resulting in a less complex microbial composition [30-32]. Our feasibility study demonstrated a high compliance, with no major side effects, indicating that longer-term studies investigating daily effects of fermented vegetables on health are feasible. Our pilot study was not powered to reveal any but substantial shifts in the gut microbiome. Hence, larger studies, potentially with longer intervention periods, are required to draw firm conclusions regarding the general population. Nevertheless, our study suggests a minor increase in gut microbiome diversity in healthy, young volunteers. The biggest change on the genera level was observed in the 
two genera Prevotella and Bacteroides. The relative abundance of Bacteroides nearly doubled while the relative abundance of Prevotella decreased in a corresponding manner. Both genera are closely related and belong to the phylum Bacteroidota. An antagonistic relation has been proposed in the literature describing some Prevotella species as being associated with chronic inflammatory conditions [33].

As reflected in the beta diversity, the gut microbiome composition differed vastly between volunteers. This was to be expected, as it has been established that the composition of the gut microbiome differs between individuals and is influenced by a great number of parameters such as diet, environment, and living situation [34]. All participants were allowed to follow their individual habitual diet in addition to the interventions. Additionally, the intervention period was relatively short. For more profound and sustained effects, fermented vegetables may have to be consumed longer in order to enable establishment of the potentially induced changes. Moreover, the small sample size fosters an intra-individual variation that exceeds the inter-individual variation in gut microbial composition. The study of the impact of fermented vegetables is also limited by difficulties in standardizing the intervention, since bacterial composition will vary even in a standardized production environment.

Our pilot study suggests that an intervention with $150 \mathrm{~g}$ of different fermented vegetables per day is feasible. This is consistent with findings from other studies such as by Nielsen et al. including an intervention with $75 \mathrm{~g}$ sauerkraut per day [3] or a study by Han et al., who investigated effects of the consumption of $180 \mathrm{~g}$ kimchi per day in a study with obese women [35].

\section{Conclusions}

Fermented vegetables contain a vast variety of bacteria that differ between respective products. Therefore, a broader exposure of a microbial variety can already be achieved by consumption of a variety of different fermented vegetables. Our study suggests that intervention studies with fermented vegetables are feasible. In addition, it provides a first look at the effects of the consumption of fermented vegetables on the human gut microbiome, suggesting an increase in microbial diversity in the human gut. This study lays a foundation for further research providing insights into the health functions of fermented vegetables.

Author Contributions: Conceptualization, S.S.H. and K.B.M.; methodology, K.T., S.S.H., N.B. and K.B.M.; software, N.B.; formal analysis, N.B.; data curation, N.B. and S.S.H., writing-original draft preparation, K.T., S.S.H. and N.B.; writing-review and editing, K.T., S.S.H., N.B. and K.B.M.; visualization, N.B.; supervision, K.B.M.; project administration, S.S.H., funding acquisition, S.S.H. All authors have read and agreed to the published version of the manuscript.

Funding: This research was funded by Forschungskommission, Medical Faculty, Albert-LudwigsUniversity Freiburg, grant number: ULL1174/18.

Institutional Review Board Statement: This study was conducted in accordance with the Declaration of Helsinki, and approved by the Ethics Committee of Albert-Ludwigs-University Freiburg (protocol code 232/18, date of approval: 25 September 2018).

Informed Consent Statement: Informed consent was obtained from all subjects involved in this study.

Data Availability Statement: The data presented in this study are available on request from the corresponding author.

Acknowledgments: We thank the team of the ZIEL Core Facility Microbiome, TUM, Freising, Germany, for sequencing the fermented vegetables. Additionally, we thank the team of the Next Generation Sequencing Core Facility (NGS-CF),University Medical Center Freiburg, Germany, for sequencing the stool samples. We acknowledge support by the Open Access Publication Fund of the University of Freiburg.

Conflicts of Interest: The authors declare no conflict of interest. 


\section{Appendix A}

Table A1. Compositional details of the fermented vegetables used in this study.

\begin{tabular}{|c|c|c|}
\hline $\begin{array}{l}\text { Type of Fermented } \\
\text { Vegetable }\end{array}$ & Ingredients & Manufacturer \\
\hline Cauliflower & $\begin{array}{l}\text { Cauliflower }(96 \%), \text { natural sea salt, } \\
\text { fresh turmeric, spices } \\
\text { (turmeric, cumin) }\end{array}$ & $\begin{array}{l}\text { completeorganics GmbH, } \\
\text { Munich, Germany }\end{array}$ \\
\hline Pink Sauerkraut & $\begin{array}{l}\text { White cabbage }(72 \%) \text {, red cabbage, } \\
\text { natural sea salt, cranberries (dried) }\end{array}$ & $\begin{array}{l}\text { completeorganics } \mathrm{GmbH} \\
\text { Munich, Germany }\end{array}$ \\
\hline Carrots & $\begin{array}{c}\text { Carrots (96\%), natural sea salt, } \\
\text { ginger (fresh) }\end{array}$ & $\begin{array}{l}\text { completeorganics } \mathrm{GmbH}, \\
\text { Munich, Germany }\end{array}$ \\
\hline Beet Root & $\begin{array}{l}\text { Beetroot (93\%), horseradish, } \\
\text { natural sea salt, goji berries (dried) }\end{array}$ & $\begin{array}{l}\text { completeorganics } \mathrm{GmbH} \\
\text { Munich, Germany }\end{array}$ \\
\hline Daikon Kimchi & $\begin{array}{l}\text { Radish }(84 \%) \text {, carrots, red peppers, } \\
\text { natural sea salt, spices } \\
\text { (ginger, chili) }\end{array}$ & $\begin{array}{l}\text { completeorganics GmbH, } \\
\text { Munich, Germany }\end{array}$ \\
\hline $\begin{array}{l}\text { Traditional } \\
\text { Sauerkraut }\end{array}$ & $\begin{array}{l}\text { White cabbage, sea salt, lactic } \\
\text { acid bacteria }\end{array}$ & $\begin{array}{c}\text { Eden Frischkost-(L+)-Sauerkraut, } \\
\text { Radolfszell, Germany }\end{array}$ \\
\hline
\end{tabular}

\section{References}

1. Marco, M.L.; Heeney, D.; Binda, S.; Cifelli, C.J.; Cotter, P.D.; Foligne, B.; Gänzle, M.; Kort, R.; Pasin, G.; Pihlanto, A.; et al. Health benefits of fermented foods: Microbiota and beyond. Curr. Opin. Biotechnol. 2017, 44, 94-102. [CrossRef] [PubMed]

2. Dimidi, E.; Cox, S.R.; Rossi, M.; Whelan, K. Fermented Foods: Definitions and Characteristics, Impact on the Gut Microbiota and Effects on Gastrointestinal Health and Disease. Nutrients 2019, 11, 1806. [CrossRef] [PubMed]

3. Nielsen, E.S.; Garnås, E.; Jensen, K.J.; Hansen, L.H.; Olsen, P.S.; Ritz, C.; Krych, L.; Nielsen, D.S. Lacto-fermented sauerkraut improves symptoms in IBS patients independent of product pasteurisation-A pilot study. Food Funct. 2018, 9, 5323-5335. [CrossRef] [PubMed]

4. Septembre-Malaterre, A.; Remize, F.; Poucheret, P. Fruits and vegetables, as a source of nutritional compounds and phytochemicals: Changes in bioactive compounds during lactic fermentation. Food Res. Int. 2018, 104, 86-99. [CrossRef]

5. Jung, J.Y.; Lee, S.H.; Kim, J.M.; Park, M.S.; Bae, J.-W.; Hahn, Y.; Madsen, E.L.; Jeon, C.O. Metagenomic Analysis of Kimchi, a Traditional Korean Fermented Food. Appl. Environ. Microbiol. 2011, 77, 2264-2274. [CrossRef]

6. Zabat, M.A.; Sano, W.H.; Wurster, J.I.; Cabral, D.J.; Belenky, P. Microbial Community Analysis of Sauerkraut Fermentation Reveals a Stable and Rapidly Established Community. Foods 2018, 7, 77. [CrossRef]

7. Rezac, S.; Kok, C.R.; Heermann, M.; Hutkins, R. Fermented Foods as a Dietary Source of Live Organisms. Front. Microbiol. 2018, 9 , 1785. [CrossRef]

8. Zmora, N.; Suez, J.; Elinav, E. You are what you eat: Diet, health and the gut microbiota. Nat. Rev. Gastroenterol. Hepatol. 2019, 16, 35-56. [CrossRef]

9. Müller, M.; Canfora, E.E.; Blaak, E.E. Gastrointestinal Transit Time, Glucose Homeostasis and Metabolic Health: Modulation by Dietary Fibers. Nutrients 2018, 10, 275. [CrossRef]

10. Roager, H.M.; Hansen, L.B.S.; Bahl, M.I.; Frandsen, H.L.; Carvalho, V.; Gøbel, R.J.; Dalgaard, M.D.; Plichta, D.R.; Sparholt, M.H.; Vestergaard, H.; et al. Colonic transit time is related to bacterial metabolism and mucosal turnover in the gut. Nat. Microbiol. 2016, 1, 16093. [CrossRef]

11. Koopman, M.; El Aidy, S. Depressed gut? The microbiota-diet-inflammation trialogue in depression. Curr. Opin. Psychiatry 2017, 30, 369-377. [CrossRef]

12. Gevers, D.; Kugathasan, S.; Denson, L.A.; Vázquez-Baeza, Y.; Van Treuren, W.; Ren, B.; Schwager, E.; Knights, D.; Song, S.J.; Yassour, M.; et al. The Treatment-Naive Microbiome in New-Onset Crohn's Disease. Cell Host Microbe 2014, 15, 382-392. [CrossRef]

13. Arrieta, M.-C.; Stiemsma, L.T.; Dimitriu, P.A.; Thorson, L.; Russell, S.; Yurist-Doutsch, S.; Kuzeljevic, B.; Gold, M.J.; Britton, H.M.; Lefebvre, D.L.; et al. Early infancy microbial and metabolic alterations affect risk of childhood asthma. Sci. Transl. Med. 2015, 7, 307ra152. [CrossRef]

14. Greenblum, S.; Turnbaugh, P.J.; Borenstein, E. Metagenomic systems biology of the human gut microbiome reveals topological shifts associated with obesity and inflammatory bowel disease. Proc. Natl. Acad. Sci. USA 2011, 109, 594-599. [CrossRef]

15. Reitmeier, S.; Kiessling, S.; Neuhaus, K.; Haller, D. Comparing Circadian Rhythmicity in the Human Gut Microbiome. STAR Protoc. 2020, 1, 100148. [CrossRef]

16. Haring, E.; Andrieux, G.; Uhl, F.M.; Krausz, M.; Proietti, M.; Sauer, B.; Esser, P.R.; Martin, S.F.; Pfeifer, D.; Schmitt-Graeff, A.; et al. Therapeutic targeting of endoplasmic reticulum stress in acute graft-versus-host disease. Haematologica 2021, 4, $2782-2858$. [CrossRef] 
17. Norona, J.; Apostolova, P.; Schmidt, D.; Ihlemann, R.; Reischmann, N.; Taylor, G.; Köhler, N.; De Heer, J.; Heeg, S.; Andrieux, G.; et al. Glucagon-like peptide 2 for intestinal stem cell and Paneth cell repair during graft-versus-host disease in mice and humans. Blood 2020, 136, 1442-1455. [CrossRef]

18. Haring, E.; Uhl, F.M.; Andrieux, G.; Proietti, M.; Bulashevska, A.; Sauer, B.; Braun, L.M.; Gomez, E.D.V.; Esser, P.R.; Martin, S.F.; et al. Bile acids regulate intestinal antigen presentation and reduce graft-versus-host disease without impairing the graft-versus-leukemia effect. Haematologica 2020, 106, 2131-2146. [CrossRef]

19. Kozich, J.J.; Westcott, S.L.; Baxter, N.T.; Highlander, S.K.; Schloss, P.D. Development of a dual-index sequencing strategy and curation pipeline for analyzing amplicon sequence data on the MiSeq Illumina sequencing platform. Appl. Environ. Microbiol. 2013, 79, 5112-5120. [CrossRef]

20. R Core Team. R: A Language and Environment for Statistical Computing; R Foundation for Statistical Computing: Vienna, Austria, 2020.

21. Callahan, B.J.; Mcmurdie, P.J.; Rosen, M.J.; Han, A.W.; Johnson, A.J.A.; Holmes, S.P. DADA 2 : High-resolution sample inference from Illumina amplicon data. Nat. Methods 2016, 13, 581-583. [CrossRef]

22. Zheng, J.; Wittouck, S.; Salvetti, E.; Franz, C.M.A.P.; Harris, H.M.B.; Mattarelli, P.; O’Toole, P.W.; Pot, B.; Vandamme, P.; Walter, J.; et al. A taxonomic note on the genus Lactobacillus: Description of 23 novel genera, emended description of the genus Lactobacillus Beijerinck 1901, and union of Lactobacillaceae and Leuconostocaceae. Int. J. Syst. Evol. Microbiol. 2020, 70, $2782-2858$. [CrossRef]

23. McMurdie, P.J.; Holmes, S. phyloseq: An R package for reproducible interactive analysis and graphics of microbiome census data. PLOS ONE 2013, 8, e61217. [CrossRef]

24. Gustaw, K.; Niedźwiedź, I.; Rachwał, K.; Polak-Berecka, M. New Insight into Bacterial Interaction with the Matrix of Plant-Based Fermented Foods. Foods 2021, 10, 1603. [CrossRef]

25. Porto, M.C.W.; Kuniyoshi, T.M.; Azevedo, P.O.S.; Vitolo, M.; Oliveira, R.P.S. Pediococcus spp.: An important genus of lactic acid bacteria and pediocin producers. Biotechnol. Adv. 2017, 35, 361-374. [CrossRef]

26. Kavitake, D.; Devi, P.B.; Shetty, P.H. Overview of exopolysaccharides produced by Weissella genus-A review. Int. J. Biol. Macromol. 2020, 164, 2964-2973. [CrossRef]

27. Hill, C.; Guarner, F.; Reid, G.; Gibson, G.R.; Merenstein, D.J.; Pot, B.; Morelli, L.; Canani, R.B.; Flint, H.J.; Salminen, S.; et al. Expert consensus document: The International Scientific Association for Probiotics and Prebiotics consensus statement on the scope and appropriate use of the term probiotic. Nat. Rev. Gastroenterol. Hepatol. 2014, 11, 506-514. [CrossRef]

28. Volokh, O.; Klimenko, N.; Berezhnaya, Y.; Tyakht, A.; Nesterova, P.; Popenko, A.; Alexeev, D. Human Gut Microbiome Response Induced by Fermented Dairy Product Intake in Healthy Volunteers. Nutrients 2019, 11, 547. [CrossRef]

29. Stiemsma, L.T.; Nakamura, R.E.; Nguyen, J.G.; Michels, K.B. Does Consumption of Fermented Foods Modify the Human Gut Microbiota? J. Nutr. 2020, 150, 1680-1692. [CrossRef]

30. Kwak, H.; Park, S.; Kim, D. Biostabilization of Kefir with a Nonlactose-Fermenting Yeast. J. Dairy Sci. 1996, 79, 937-942. [CrossRef]

31. Marsh, A.J.; O'Sullivan, O.; Hill, C.; Ross, R.; Cotter, P.D. Sequencing-Based Analysis of the Bacterial and Fungal Composition of Kefir Grains and Milks from Multiple Sources. PLoS ONE 2013, 8, e69371. [CrossRef]

32. Bourrie, B.C.T.; Willing, B.P.; Cotter, P.D. The Microbiota and Health Promoting Characteristics of the Fermented Beverage Kefir. Front. Microbiol. 2016, 7, 647. [CrossRef]

33. Ley, R.E. Prevotella in the gut: Choose carefully. Nat. Rev. Gastroenterol. Hepatol. 2016, 13, 69-70. [CrossRef] [PubMed]

34. Conlon, M.A.; Bird, A.R. The Impact of Diet and Lifestyle on Gut Microbiota and Human Health. Nutrients 2014, 7, 17-44. [CrossRef] [PubMed]

35. Han, K.; Bose, S.; Wang, J.-H.; Kim, B.-S.; Kim, M.J.; Kim, E.-J.; Kim, H. Contrasting effects of fresh and fermented kimchi consumption on gut microbiota composition and gene expression related to metabolic syndrome in obese Korean women. Mol. Nutr. Food Res. 2015, 59, 1004-1008. [CrossRef] [PubMed] 